\title{
ON THE DIMENSION OF INJECTIVE BANACH SPACES
}

\author{
S. ARGYROS
}

\begin{abstract}
The purpose of this note is to give an affirmative answer, assuming the generalized continuum hypothesis, to a problem of $\mathbf{H}$. Rosenthal on the cardinality of the dimension on injective Banach spaces.
\end{abstract}

The problem in question is contained in [4, Problem 7.a]; in this connection we prove the following result.

Theorem 1. Assume the G.C.H. If $X$ is an infinite dimensional injective Banach space with $\operatorname{dim} X=\alpha$, then $\alpha^{\omega}=\alpha$.

We start with some preliminaries.

We denote cardinals by $\alpha, \beta$; $\omega$ denotes the cardinality of natural numbers. We denote by $\alpha^{\omega}$ the cardinality of the family of countable subsets of $\alpha$. For a cardinal $\alpha$, we denote by $\operatorname{cf}(\alpha)$ the least cardinal $\beta$ such that $\alpha$ is the cardinal sum of $\beta$ many cardinals, each smaller than $\alpha$. A cardinal $\alpha$ is regular if $\alpha=\operatorname{cf}(\alpha)$, and singular if $\operatorname{cf}(\alpha)<\alpha$. The least cardinal strictly greater than $\beta$ is denoted by $\beta^{+}$. The cardinality of a set $A$ is denoted by $|A|$. The generalised continuum hypothesis (G.C.H.) is the statement that $\alpha^{+}=2^{\alpha}$ for all infinite cardinals $\alpha$.

A real Banach space $X$ is injective if for every Banach space $Y$ and every bounded linear isomorphism $T: X \rightarrow Y$, there is a bounded linear projection $P: Y \rightarrow T(X)$. If $\Gamma$ is a set, we denote by $l^{1}(\Gamma)$ the Banach space of real-valued functions on $\Gamma$ which are absolutely summable. If $X$ is a Banach space we denote with $\operatorname{dim} X$ the least cardinal $\alpha$ such that there is a family $F=\left\{x_{\xi}: \xi<\alpha\right\}$ of elements of $X$ with the property that $X$ is the closed linear span of $F$.

Lemma 2. Let $X$ be an injective Banach space with $\operatorname{dim} X=\alpha$. Then $l^{1}(\alpha)$ is isomorphic to a subspace of $X^{*}$.

Proof. Since $X$ is a complemented subspace of $C(S)$ for some compact space $S$, $X^{*}$ is a complemented subspace of $L^{1}(\lambda)$ for some measure $\lambda$. So the conclusion is a direct consequence of Theorem 2.5 of [3].

Proof of Theorem 1. Let us assume that the conclusion is false. Then there is an injective Banach space $X$ with $\operatorname{dim} X=\alpha$ and $\alpha^{\omega}>\alpha$. Under the G.C.H., $\alpha^{\omega}>\alpha$ means that $\operatorname{cf}(\alpha)=\omega$ and since $l^{\infty}(\mathbf{N})$ is isomorphic to a subspace of $X$ [5] it follows that $\alpha>\operatorname{cf}(\alpha)$.

We choose a sequence $\left\{\alpha_{\eta}: \eta<\omega\right\}$ of regular cardinals such that $\alpha_{1}=\omega^{+}$, $\alpha_{\eta+1}>2^{\alpha_{\eta}}$ and $\Sigma_{\eta<\omega} \alpha_{\eta}=\alpha$.

Received by the editors December 7, 1978 and, in revised form, March 16, 1979.

AMS (MOS) subject classifications (1970). Primary 46B05; Secondary 06A40. 
From Lemma 2 there is a family $\left\{e_{\xi}: \xi<\alpha\right\}$ of elements of the unit ball of $X^{*}$ equivalent to the canonical basis for $l^{1}(\alpha)$.

Let, also, $\left\{x_{\xi}: \xi<\alpha\right\}$ be a norm dense subset of $X$. Using finite induction we choose a family $\left\{A_{\eta}: \eta<\omega\right\}$ of subsets of $\alpha$ such that:

(i) $A_{\eta} \subset\left\{\xi: \alpha_{\eta}<\xi<\alpha_{\eta+1}\right\}$,

(ii) $\left|A_{\eta}\right|>2$, and

(iii) for $\eta<\omega$ and $\xi_{1}, \xi_{2} \in A_{\eta}$

$$
e_{\xi_{1}}\left(x_{\xi}\right)=e_{\xi_{2}}\left(x_{\xi}\right) \text { for all } \xi<\alpha_{\eta} \text {. }
$$

For every $\eta<\omega$ we choose $\xi_{1}^{\eta} \neq \xi_{2}^{\eta}$ elements of $A_{\eta}$, and we set $e_{\eta}=e_{\xi_{1}}-e_{\xi_{2}}$. Then the sequence $\left\{e_{\eta}: \eta<\omega\right\}$ converges weak* to $0 \in X^{*}$, and since $X$ is injective, $\left\{e_{\eta}: \eta<\omega\right\}$ is in fact weakly convergent [2]. On the other hand, $\left\{e_{\eta}\right.$ : $\eta<\omega\}$ is equivalent to the usual basis for $l^{1}(\mathbf{N})$, a contradiction.

REMARK 1. As the referee has remarked, the proof shows immediately the following more general statement:

If $X$ is an $\mathfrak{L}_{\infty}$ Grothendieck space, then under the G.C.H. we have $(\operatorname{dim} X)^{\omega}=$ $\operatorname{dim} X$. (Recall that a Banach space $X$ is a Grothendieck space if every sequence in $X^{*}$ which is weak* convergent necessarily converges weakly.)

REMARK 2. We do not know what happens without any set-theoretical assumption. In this direction we proved in [1] the following.

THEOREM A. If $X$ is an injective Banach space in which each weakly compact subset is separable and $\operatorname{dim} X=\alpha$ then $\alpha^{\omega}=\alpha$.

THEOREM B. Let $\alpha$ be a cardinal and $X$ be an injective Banach space such that $l^{1}(\alpha)$ is isomorphic to a subspace of $X$. Then $X$ contains isomorphically a copy of $l^{1}\left(\alpha^{\omega}\right)$.

\section{REFERENCES}

1. S. Argyros, Weak compactness in $L^{1}(\lambda)$ and injective Banach spaces (to appear).

2. A. Grothendieck, Sur les applications lineaires faiblement compactes d' espaces du type $C(K), C a n a d$. J. Math. 5 (1953), 129-173.

3. R. Haydon, On dual $L^{1}$-spaces and injective bidual Banach spaces, Israel J. Math. 31 (1979), $142-152$.

4. H. P. Rosenthal, On injective Banach spaces $L^{\infty}(\mu)$ for finite measures $\mu$, Acta Math. 124 (1970), 205-247.

5. On relatively disjoint families of measures with some applications to Banach spaces theory, Studia Math. 37 (1970), 13-36.

Chair I of Mathematical Analysis, Athens University, Panepistmmopolis, Athens 621, Greece 